\section{Validade de construto de uma versão em português do Female Sexual Function Index}

\author{
Construct validity of a Portuguese version \\ of the Female Sexual Function Index
}

\footnotetext{
${ }^{1}$ Centro de Ciências Biológicas e da Saúde, Universidade Federal de São Carlos, São Carlos, Brasil. 2 Faculdade de Medicina de Ribeirão Preto, Universidade de São Paulo, Ribeirão Preto, Brasil.

Correspondência E. M. Vieira

Departamento de Medicina Social, Faculdade de Medicina de Ribeirão Preto, Universidade de São Paulo. $A v$. dos Bandeirantes 3900 , 2o andar, Ribeirão Preto, $S P$ 14049-900, Brasil. bmeloni@fmrp.usp.br
}

\begin{abstract}
This study aimed to evaluate the construct validity of a version of the Female Sexual Function Index (FSFI). The version was inserted into a multidimensional questionnaire and applied face-to-face to 235 sterilized women. Non-response rate was $1.7 \%$. The sample proved to be highly homogeneous, with low income and education. Cronbach's alpha of 0.948 indicated high internal consistency. Factor analysis showed that the instrument was measuring four factors: desire/arousal, lubrication, orgasm/satisfaction, and pain. This may reflect characteristics of the instrument itself, female sexual response, or even the sample's cultural peculiarities, which can affect understanding of the questions or concepts with which the instrument deals. This study emphasizes the need for further research in the general population to determine the psychometric properties of the FSFI, such as its factor composition and definition of appropriate cut-off points for the Brazilian population.
\end{abstract}

Sexuality; Questionnaires; Women's Health
Rodolfo de Carvalho Pacagnella 1

Edson Zangiacomi Martinez 2

Elisabeth Meloni Vieira 2

\section{Introdução}

Alguns autores sugerem que as disfunções sexuais femininas chegam a atingir mais de $40 \%$ das mulheres 1 . Com o crescente interesse da comunidade científica pelos problemas sexuais das mulheres tem-se, então, buscado consenso diagnóstico sobre o tema. Diferentemente dos manuais nosológicos, como o manual de Classificação Internacional de Doenças, 10a revisão (CID-10) e o Diagnostic and Statistical Manual of Mental Disorders (DSM-IV), as atuais propostas diagnósticas levam em consideração novos modelos não lineares da resposta sexual feminina e classificam os problemas sexuais de maneira mais abrangente, pois reconhecem e incorporam, por exemplo, algumas das dimensões não genitais e subjetivas da excitação feminina ${ }^{2}$ e não apenas a etiologia das disfunções.

Nesse sentido, ao se incorporar essas nuances da resposta sexual feminina na classificação das disfunções sexuais coloca-se a necessidade de se desenvolver instrumentos capazes de avaliar essas questões de maneira adequada. O recente consenso sobre a classificação das disfunções femininas recomenda o processo de padronização e validação de questionários para a avaliação da função e resposta sexual das mulheres 2 . Segundo alguns autores, considerando os fatores contextuais e a característica subjetiva da resposta sexual feminina, os instrumentos com maior possibilidade de avaliar as suas questões 
objetivas e subjetivas seriam os questionários auto-aplicados que avaliam vários domínios no campo da sexualidade e apresentam alto grau de confiabilidade e validade ${ }^{3}$. Estes seriam mais adequados à avaliação das disfunções sexuais femininas que os diários de eventos (event logs), pois são delineados para utilizar a escala ordinal e de intervalo com a capacidade de detectar diferenças sutis em um nível sofisticado de medida 3 . Os questionários teriam a capacidade de traduzir informações subjetivas em dados quantificáveis e analisáveis de forma global ou específica.

Nesse contexto, tem-se buscado o desenvolvimento de questionários com a capacidade de avaliar múltiplos aspectos da resposta sexual das mulheres. Alguns dos instrumentos validados mais estudados atualmente são o Brief Sexual Function Index for Women, o The Derogatis Interview for Sexual Functioning e o Female Sexual Function Index (FSFI) 4.

Desses instrumentos, o FSFI foi o mais recentemente desenvolvido e está em consonância com os novos modelos da resposta sexual feminina. Esse questionário, proposto por Rosen et al. 4 no ano de 2000 nos Estados Unidos e validado por outros autores 5,6, foi projetado para ser um instrumento de avaliação em estudos epidemiológicos que respeita a natureza multidimensional da função sexual feminina. Segundo Rosen et al. 4 , problemas que afetem um domínio da resposta sexual humana podem interagir com outras desordens de uma forma complexa resultando potencial viés na categorização das disfunções sexuais. Assim, há necessidade de instrumentos capazes de avaliar a relativa força da disfunção em cada domínio.

O FSFI é um questionário breve, que pode ser auto-aplicado, e que se propõe avaliar a resposta sexual feminina em seis domínios: desejo sexual, excitação sexual, lubrificação vaginal, orgasmo, satisfação sexual e dor. Para isso, apresenta 19 questões que avaliam a função sexual nas últimas quatro semanas. Para cada questão existe um padrão de resposta cujas opções recebem pontuação de 0 a 5 de forma crescente em relação à presença da função questionada. Apenas nas questões sobre dor a pontuação é definida de forma invertida. Um escore total é apresentado ao final da aplicação, resultado da soma dos escores de cada domínio multiplicada por um fator que homogeneíza a influência de cada domínio no escore total (Figura 1). Muito embora o instrumento não tenha a capacidade de discriminar a fase da resposta alterada, a partir de um ponto de corte do escore total (definido como 26 para a população de origem do instrumento) seria possível discriminar entre as populações com maior e menor risco de apresentar disfunção sexual, sendo que valores iguais ou abaixo desse ponto indicariam disfunção sexual 6 .

É nesse sentido que o FSFI congrega as características de ser prático para aplicação em estudos populacionais, transformar medidas subjetivas em dados objetivos, quantificáveis e analisáveis e avaliar a força relativa de cada domínio ou componente da resposta sexual feminina. Outras vantagens desse questionário residem no fato de ser um índice bem estudado, validado 6 e com capacidade de avaliar intervenções terapêuticas 7 . Ademais, o FSFI tem sido submetido à adaptação para outros idiomas além do inglês - inclusive para a língua espanhola e para o contexto cultural da América Latina - e tem sido validado nessas línguas 8,9.

No Brasil, existem poucos questionários desenvolvidos para avaliar a função sexual e não há questionários desenvolvidos em língua estrangeira que tenham sido adequadamente adaptados e validados para o contexto cultural brasileiro. Alguns estudos 10,11,12 utilizam o Golombok Rust Inventory of Sexual Satisfaction (GRISS) em pesquisas na população brasileira, no entanto sem a adaptação adequada do mesmo para esse contexto.

Outros autores 13 apresentaram um instrumento com o objetivo de avaliar ampla e detalhadamente hábitos, tendências e práticas sexuais da população brasileira e propõem um questionário simplificado para detectar possíveis portadores de dificuldades sexuais de diferentes níveis 14 . Porém, com a existência de número cada vez maior de estudos multicêntricos é adequada a adaptação de um mesmo instrumento para contextos culturais diversos, de forma a possibilitar comparações entre grupos populacionais. Assim, seria oportuno considerar a utilização do FSFI fora do contexto da língua inglesa, pois, o instrumento apresenta características que apóiam seu uso em situações diversas.

Nesse sentido, Pacagnella et al. 15 realizaram a adaptação transcultural desse instrumento seguindo rígido padrão metodológico a fim de preservar as características de medida do questionário para a utilização entre as mulheres brasileiras. Alguns autores referem que o próprio rigor desse processo de adaptação transcultural de um instrumento de medida garante a validade do instrumento na população de destino, porém, existem poucas garantias de que as propriedades psicométricas do instrumento se mantenham 16 . Outros autores apontam que embora as propriedades psicométricas do instrumento original já tenham sido estabelecidas, é necessário determiná-las na versão da língua de destino 17 .

A validação de um instrumento permite identificar se o instrumento está realmente medindo 
Este questionário pergunta sobre sua vida sexual durante as últimas 4 semanas. Por favor, responda às questões de forma mais honesta e clara possível. Suas respostas serão mantidas em absoluto sigilo. Para responder as questões use as seguintes definições: Atividade sexual pode incluir afagos, carícias preliminares, masturbação ("punheta"/"siririca") e ato sexual. Ato sexual é definido quando há penetração (entrada) do pênis na vagina.

Estímulo sexual inclui situações como carícias preliminares com um parceiro, auto-estimulação (masturbação) ou fantasia sexual (pensamentos) ASSINALE APENAS UMA ALTERNATIVA POR PERGUNTA

Desejo sexual ou interesse sexual é um sentimento que inclui querer ter atividade sexual, sentir-se receptiva a uma iniciativa sexual de um parceiro(a) e pensar ou fantasiar sobre sexo.

Excitação sexual é uma sensação que inclui aspectos físicos e mentais. Pode incluir sensações como calor ou inchaço dos genitais, lubrificação (sentir-se molhada/"vagina molhada"/"tesão vaginal"), ou contrações musculares.

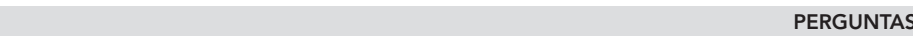

1- Nas últimas 4 semanas com que freqüência (quantas vezes) você sentiu desejo ou interesse sexual?

Padrão de resposta: $\mathrm{A}$

2- Nas últimas 4 semanas como você avalia o seu grau de desejo ou interesse sexual?

Padrão de resposta: $B$

3- Nas últimas 4 semanas, com que freqüência (quantas vezes) você se sentiu sexualmente excitada durante a atividade sexual ou ato sexual?

Padrão de resposta: $A$

4- Nas últimas 4 semanas, como você classificaria seu grau de excitação sexual durante

a atividade ou ato sexual?

Padrão de resposta: $B$

5- Nas últimas 4 semanas, como você avalia o seu grau de segurança para ficar sexualmente excitada durante a atividade sexual ou ato sexual?

Padrão de resposta: $C$

6- Nas últimas 4 semanas, com que freqüência (quantas vezes) você ficou satisfeita com sua excitação sexual durante a atividade sexual ou ato sexual?

Padrão de resposta: $\mathrm{A}$

7- Nas últimas 4 semanas, com que freqüência (quantas vezes) você teve lubrificação vaginal (ficou com a vagina

"molhada") durante a atividade sexual ou ato sexual?
8- Nas últimas 4 semanas, como você avalia sua dificuldade em ter lubrificação vaginal (ficar com a vagin "molhada") durante $o$ ato sexual ou atividades sexuais?

\section{Padrão de resposta: D}

9- Nas últimas 4 semanas, com que freqüência (quantas vezes) você manteve a lubrificação vaginal (ficou com a vagina "molhada") até o final da atividade ou ato sexual?

\section{Padrão de resposta: $A$}

10- Nas últimas 4 semanas, qual foi sua dificuldade em manter a lubrificação vaginal (vagina "molhada") até o final da atividade ou ato sexual?

Padrão de resposta: $D$

11- Nas últimas 4 semanas, quando teve estímulo sexual ou ato sexual, com que freqüência (quantas vezes) você atingiu o orgasmo ("gozou")?

Padrão de resposta: $\mathrm{A}$

12 - Nas últimas 4 semanas, quando você teve estímulo sexual ou ato sexual, qual foi sua dificuldade em você atingir o orgasmo "(clímax/"gozou")"?

Padrão de resposta: D

13- Nas últimas 4 semanas, o quanto você ficou satisfeita com sua capacidade de atingir o orgasmo ("gozar") durante atividade ou ato sexual?

Padrão de resposta: $\mathrm{E}$
14- Nas últimas 4 semanas, o quanto você esteve satisfeita com a proximidade emocional entre você e seu parceiro(a) durante a atividade sexual?

\section{Padrão de resposta: $E$}

15- Nas últimas 4 semanas, o quanto você esteve satisfeita com o relacionamento sexual entre você e seu parceiro(a)?

Padrão de resposta: $\mathrm{E}$

16- Nas últimas 4 semanas, o quanto você esteve satisfeita com sua vida sexual de um modo geral?

\section{Padrão de resposta: $\mathrm{E}$}

17- Nas últimas 4 semanas, com que freqüência (quantas vezes) você sentiu desconforto ou dor durante a penetração vaginal?

\section{Padrão de resposta: A}

18- Nas últimas 4 semanas, com que freqüência (quantas vezes) você sentiu desconforto ou dor após a penetração vaginal?

Padrão de resposta: $\mathrm{A}$

19- Nas últimas 4 semanas, como você classificaria seu grau de desconforto ou dor durante ou após a penetração vaginal?

Padrão de resposta: $B$

OBS- Nas questões 1 e 2, não há a alternativa "Sem atividade sexual"

Padrão de resposta: $A$

\section{RESPOSTAS}

1. Sem atividade sexual.

2. Quase sempre ou sempre.

3. A maioria das vezes (mais do

que a metade do tempo)

4. Algumas vezes (cerca de

metade do tempo)

5. Poucas vezes (menos da

metade do tempo)

6. Quase nunca ou nunca.
1. Sem atividade sexual.

2. Muito alto.

3. Alto.

4. Moderado

5. Baixo.

6. Muito baixo ou absolutamente nenhum.

\section{C}

1. Sem atividade sexual.

2. Segurança muito alta. 3. Segurança alta.

4. Segurança moderada

5. Segurança baixa.

6. Segurança muito baixa ou sem segurança.
1. Sem atividade sexual.

2. Extremamente difícil ou impossível.

3. Muito difíci 4. Difícil.

5. Ligeiramente difícil. 6. Nada difícil.
1. Sem atividade sexual.

2. Muito satisfeita.

3. Moderadamente satisfeita.

4. Quase igualmente satisfeita e insatisfeita.

5. Moderadamente insatisfeita. 6. Muito insatisfeita.

\begin{tabular}{|c|c|c|c|c|c|}
\hline \multicolumn{6}{|c|}{ ESCORES DE AVALIAÇÃO DO FSFI 17} \\
\hline Domínio & Questões & Variação do escore & Fator de multiplicação & Escore mínimo & Escore máximo \\
\hline Desejo & 1,2 & $1-5$ & 0,6 & 1,2 & 6,0 \\
\hline Excitação & $3,4,5,6$ & $0-5$ & 0,3 & 0,0 & 6,0 \\
\hline Lubrificação & $7,8,9,10$ & $0-5$ & 0,3 & 0,0 & 6,0 \\
\hline Orgasmo & $11,12,13$ & $0-5$ & 0,4 & 0,0 & 6,0 \\
\hline Satisfação & $14,15,16$ & 0 (ou 1$)-5$ * & 0,4 & 0,8 & 6,0 \\
\hline Dor & $17,18,19$ & $0-5$ & 0,4 & 0,0 & 6,0 \\
\hline
\end{tabular}

* Questão 14 varia de 0-5; questões 15 e 16 variam de 1-5. 
o que se espera medir, para tanto ele terá validade quando congregar alguns atributos como validade de critério, de conteúdo e de construto ${ }^{17}$. A validade de construto diz respeito a um processo pelo qual a medida se correlaciona com as variáveis de maneira consistente com a teoria $17,18,19,20$

Dessa forma, o presente estudo teve por objetivo analisar a validade de construto da versão em português do instrumento FSFI para a aplicação no contexto brasileiro, como parte do processo de validação e definição das propriedades psicométricas do questionário.

\section{Método}

\section{Desenho e contexto de estudo}

A versão em português do FSFI foi testada em um estudo transversal componente de um projeto de Vieira 21, que teve por objetivo geral avaliar a satisfação com métodos cirúrgicos de esterilização após a implantação da Lei $n^{\circ}$. 9.263. Este estudo foi previamente aprovado pelo Comitê de Ética em Pesquisa do Centro de Saúde Escola da Faculdade de Medicina de Ribeirão Preto da Universidade de São Paulo (processo no ${ }^{\circ}$ 0066/03). A versão do FSFI foi inserida ao final de um questionário multidimensional que continha questões relacionadas às características sócio-demográficas e reprodutivas das mulheres. Uma descrição do instrumento está apresentada na Figura 1.

Estratégias de seleção e tamanho da amostra

A seleção das participantes foi feita a partir da população de mulheres que realizaram voluntariamente a esterilização cirúrgica pelo Sistema Único de Saúde (SUS) em Ribeirão Preto, São Paulo, Brasil, no período de fevereiro de 2000 a dezembro de 2004. Nesse período foram realizadas 1.824 cirurgias de esterilização feminina. Assim, considerando-se a fórmula de amostragem para população finita e os objetivos do estudo principal, realizou-se um sorteio baseado em procedimento de amostragem sistemática que resultou uma amostra de 235 casos, suficiente para a análise de validade de construto ${ }^{18}$.

Como critério de inclusão foram consideradas na análise apenas as mulheres que já haviam tido relação sexual uma vez na vida. Como critérios de exclusão, além da recusa em responder o questionário, foram excluídas da análise da função sexual todas as mulheres que não responderam a pelo menos uma questão do instrumento de avaliação da função sexual.

\section{Coleta de dados}

Apesar do FSFI ter sido desenvolvido para ser auto-aplicado, considerou-se necessária sua aplicação face a face por entrevistadoras treinadas em virtude das possíveis características de escolaridade das mulheres da amostra, todas usuárias do SUS. Para a aplicação dos questionários foram selecionadas cinco entrevistadoras com Ensino Superior completo ou em andamento, boa comunicação, experiência anterior com entrevistas e sem vínculo com os programas de saúde do município. Todas foram intensamente treinadas para a tarefa.

As entrevistas foram realizadas entre os meses de agosto e dezembro de 2005. Todas as entrevistadoras foram orientadas a fazer até três tentativas de visitas, com dias e horários alternados para aumentar a possibilidade das entrevistadas serem encontradas em seus domicílios. As mulheres que não foram encontradas até a terceira visita ou em cujos casos se observaram mudança de endereço, endereço inconsistente ou morte foram substituídas por outras que realizaram a laqueadura tubária no mesmo ano que aquelas que não foram encontradas.

\section{Análise de dados}

Os dados dos questionários foram, então, digitados no programa Epi Info (Centers for Disease Control and Prevention, Atlanta, Estados Unidos). Para a análise utilizou-se o pacote estatístico para computador SAS versão 9 (SAS Inst., Cary, Estados Unidos) como ferramenta na elaboração de tabelas e testes estatísticos. Foram utilizados o coeficiente de correlação de Pearson para a análise de correlação inter-domínios, a análise fatorial exploratória com rotação varimax, para melhor interpretabilidade dos fatores e o coeficiente alfa de Cronbach para a mensuração da consistência interna do FSFI.

A análise fatorial é uma ferramenta multivariada de análise de dados, que permite a identificação de fatores dentro do conjunto dos itens do instrumento de avaliação, indicando os mais relacionados entre si. Estes fatores são variáveis latentes, ou seja, variáveis não observáveis, construídas a partir das variáveis originais. São interpretados na ordem do mais explicativo para o menos explicativo, ou seja, segundo a proporção da variabilidade geral dos dados originais por eles explicada. As cargas fatoriais, obtidas da análise, identificam o quanto cada fator está associado a cada item do instrumento e o quanto o conjunto de fatores explica a variabilidade geral dos dados originais. Os autovalores, também gerados pela 
análise fatorial, são números que refletem a importância do fator 22 .

Optou-se pela utilização da análise fatorial exploratória pois, a população de estudo apresentava particularidades quanto ao nível sócioeducacional que a diferem da população em que o instrumento original foi validado, o que motivou inclusive a aplicação do instrumento na forma de entrevistas face a face. Nesse sentido a intenção da análise fatorial exploratória foi identificar os conjuntos de fatores latentes para a população estudada. Para tanto considerou-se o critério dos autovalores mínimos (minimum eigenvalue criterion) para escolha do número de fatores baseado na escolha dos fatores cujos autovalores são maiores que 1 .

Na avaliação da consistência interna, além do alfa de Cronbach total, foi realizada uma análise da correlação entre cada item e o total, que é o coeficiente de correlação entre o item específico e o indicador total depurado de sua própria contribuição. Esta correlação mede a associação do item com as outras medidas tomadas em conjunto. Ainda, foi feita uma análise do alfa de Cronbach quando cada item individualmente é desprezado, o que mostra o impacto que teria a retirada deste item do questionário.

\section{Resultados}

A amostra estudada apresentou-se bastante homogênea em suas características sócio-demográficas e educacionais. As características das mulheres entrevistadas encontram-se descritas na Tabela 1. A média de idade das participantes foi de 35,9 anos; encontravam-se em união no momento da entrevista 89,8\%. Quanto à cor, a maioria se auto-declarou branca $(57,9 \%)$. Sobre a religião que seguem, 157 mulheres disseram ser católicas $(66,8 \%)$ e $64(27,2 \%)$ declararam-se evangélicas.

A maioria das mulheres $(66,8 \%)$ possuía menos de oito anos completos de estudo, a média foi de 6,1 anos. Quanto ao grau de escolaridade, 80,5\% das mulheres possuía apenas o Ensino Fundamental sendo que cerca de $65 \%$ não o haviam completado. As entrevistadas foram categorizadas em cinco grupos conforme a classificação sócio-econômica da Associação Brasileira de Empresas de Pesquisa (Critério de classificação econômica Brasil. http://www.abep.org/codigoguia/ ABEP_CCEB.pdf, acessado em 11/Out/2005). Mais de $50 \%$ das mulheres pertenciam à categoria $\mathrm{C}$ e $32 \%$ à categoria $\mathrm{D}$. A renda média das famílias das entrevistadas foi $\mathrm{R} \$$ 854,03 mensais.

Todas as mulheres entrevistadas responderam à avaliação da função sexual, no entanto
Tabela 1

Características sócio-demográficas e educacionais das mulheres esterilizadas entre 2000 e 2004 Ribeirão Preto, São Paulo, Brasil.

\begin{tabular}{|c|c|c|}
\hline Características & $\mathbf{n}$ & $\%$ \\
\hline \multicolumn{3}{|l|}{ Idade (anos completos) } \\
\hline $25-29$ & 28 & 11,9 \\
\hline $30-34$ & 66 & 28,1 \\
\hline $35-39$ & 86 & 36,6 \\
\hline $40-44$ & 43 & 18,3 \\
\hline 45 ou mais & 12 & 5,1 \\
\hline \multicolumn{3}{|l|}{ Estado marital } \\
\hline Casadas & 119 & 50,6 \\
\hline Moram junto & 92 & 39,2 \\
\hline Solteiras & 12 & 5,1 \\
\hline Separadas & 9 & 3,8 \\
\hline Viúvas & 3 & 1,3 \\
\hline \multicolumn{3}{|l|}{ Cor } \\
\hline Branca & 136 & 57,9 \\
\hline Parda & 71 & 30,2 \\
\hline Preta & 28 & 11,9 \\
\hline \multicolumn{3}{|l|}{ Religião } \\
\hline Católica & 157 & 66,8 \\
\hline Evangélica & 64 & 27,2 \\
\hline Espírita & 6 & 2,6 \\
\hline Protestante & 1 & 0,4 \\
\hline Outras & 7 & 3,0 \\
\hline \multicolumn{3}{|l|}{ Escolaridade (anos completos) } \\
\hline $1-7$ & 153 & 66,2 \\
\hline Acima de 7 & 78 & 33,8 \\
\hline \multicolumn{3}{|l|}{ Grau de escolaridade } \\
\hline Ensino Fundamental incompleto & 152 & 65,8 \\
\hline Ensino Fundamental completo & 34 & 14,7 \\
\hline Ensino Médio incompleto & 21 & 9,1 \\
\hline Ensino Médio completo & 22 & 9,5 \\
\hline Ensino Superior completo & 2 & 0,9 \\
\hline \multicolumn{3}{|l|}{ Classificação econômica * } \\
\hline A & 5 & 2,1 \\
\hline B & 49 & 20,9 \\
\hline C & 125 & 53,2 \\
\hline $\mathrm{D}$ & 55 & 23,4 \\
\hline$E$ & 1 & 0,4 \\
\hline
\end{tabular}

* De acordo com a Associação Brasileira de Empresas de Pesquisa (Critério de Classificação Econômica Brasil. http://www.abep.org/codigoguia/ABEP_CCEB.pdf, acessado em 11/Out/2005).

quatro entrevistadas não responderam a questão referente à satisfação com o relacionamento entre ela e o parceiro, duas das quais também não responderam as questões referentes à avaliação da satisfação com a vida sexual. Todas essas mulheres não estavam coabitando com parceiros no momento da entrevista e, portanto, foram classificadas como "não unidas" na análise do esta- 
do marital. Dessa forma, obtivemos uma taxa de não resposta de $1,7 \%$.

$\mathrm{Na}$ análise de correlação observou-se significância estatística em todos os pares (Tabela 2). Os maiores índices de correlação foram observados entre orgasmo e excitação, satisfação e orgasmo, excitação e desejo, entre orgasmo e lubrificação e entre excitação e lubrificação. Também se observou menor correlação entre dor e todos os outros domínios incluindo satisfação.

Os resultados da análise fatorial com os respectivos itens, cargas fatoriais, percentual de variância explicada e autovalores estão apresentados na Tabela 3. Os itens cujas cargas fatoriais são maiores que 0,40 são mostrados em negrito. Dentre o conjunto de fatores gerados (19 fatores, ao todo), selecionaram-se aqueles com maior percentual de variância explicada. Pelo critério minimum eigenvalue criterion encontrou-se solução fatorial de quatro fatores (explicando $74,6 \%$ da variância total).

O fator 1 (52,5\% de variância explicada) agrupou os três itens do FSFI associados à satisfação, os três itens relativos ao orgasmo e o item relativo à satisfação do domínio que reúne os itens relacionados à excitação. O fator 2 (9,7\% de variância explicada) agrupou os itens dos domínios relativos ao desejo e à excitação, mostrando também uma carga fatorial relativamente alta ao item associado à freqüência no domínio relativo ao orgasmo. O fator 3 (6,7\% de variância explicada) agrupou os quatro itens do domínio relativo à lubrificação, com cargas fatoriais maiores que 0,5 , e incluiu ainda o item associado à dificuldade do domínio relativo ao orgasmo. O fator 4 (5,7\% de variância explicada) agrupou os três itens do domínio associado à dor, com respectivas cargas fatoriais relativamente grandes.

A consistência interna do FSFI foi medida pelo coeficiente alfa de Cronbach. Foi encontrado um coeficiente alfa de 0,948 . Outras informações sobre o comportamento de cada item e de cada domínio no cálculo do coeficiente alfa de Cronbach são listadas na Tabela 4 . Nota-se que os itens 17 e 19, respectivamente a freqüência e o grau da dor durante a penetração vaginal, possuem menor correlação entre o item e o total do FSFI.

$\mathrm{Na}$ Tabela 4, ainda, nota-se que a eliminação de qualquer um dos itens não implica uma significativa mudança do alfa de Cronbach.

\section{Discussão}

A população de estudo mostrou-se muito homogênea quanto às condições sócio-demográficas e educacionais, caracterizada por ser de baixa escolaridade e renda. Isso é consistente com a população de referência, mulheres submetidas à esterilização cirúrgica pelo SUS, porém diverge da população em que foi validado o instrumento original, melhor distribuída em todas as classes sociais e níveis educacionais 4 .

A publicação original do instrumento propõe sua aplicação na forma de questionário auto-aplicado 4 mas alguns atores consideram que diferenças no nível educacional e cultural podem afetar a validade desses instrumentos auto-aplicados 3,23. Assim, dadas as características da amostra estudada, a opção pelo uso do instrumento na forma de aplicação face a face mostrou-se adequada. A aplicação do questionário transcorreu de forma clara e sem dificuldades e essa forma de aplicação foi bem aceita pelas mulheres estudadas o que se reflete no baixo índice de não resposta $(1,7 \%)$.

A análise de correlação inter-domínios ofereceu informação adicional sobre a associação entre os domínios, evidenciando forte correlação entre todas as fases da resposta sexual, entre desejo e excitação, entre excitação e lubrificação e entre excitação e orgasmo além de forte corre-

Tabela 2

Análise de correlação inter-domínios do Female Sexual Function Index (FSFI).

\begin{tabular}{llcccc}
\hline & Desejo & Excitação & Lubrificação & Orgasmo & Satisfação \\
\hline Desejo & 1,0000 & & & & \\
Excitação & 0,7341 & 1,0000 & & & \\
Lubrificação & 0,4845 & 0,7170 & 1,0000 & & 1,0000 \\
Orgasmo & 0,6152 & 0,7964 & 0,7310 & 0,7665 & 0,3947 \\
Satisfação & 0,5717 & 0,6833 & 0,5873 & 0,4748 & 1,0000 \\
Dor & 0,3894 & 0,4534 & 0,5319 & & \\
\hline
\end{tabular}

Nota: $p<0,05$. 
Análise fatorial com rotação varimax do Female Sexual Function Index (FSFI).

\begin{tabular}{|c|c|c|c|c|}
\hline Domínio/Questão & Fator 1 & Fator 2 & Fator 3 & Fator 4 \\
\hline \multicolumn{5}{|l|}{ Desejo } \\
\hline 1: Freqüência & 0,337 & 0,715 & 0,061 & 0,255 \\
\hline 2: Nível & 0,200 & 0,840 & 0,106 & 0,073 \\
\hline \multicolumn{5}{|l|}{ Excitação } \\
\hline 3: Freqüência & 0,327 & 0,677 & 0,386 & 0,170 \\
\hline 4: Nível & 0,228 & 0,717 & 0,385 & 0,078 \\
\hline 5: Confiança & 0,192 & 0,609 & 0,355 & 0,176 \\
\hline 6: Satisfação & 0,546 & 0,515 & 0,360 & 0,188 \\
\hline \multicolumn{5}{|l|}{ Lubrificação } \\
\hline 7: Freqüência & 0,383 & 0,311 & 0,592 & 0,239 \\
\hline 8: Dificuldade & 0,178 & 0,210 & 0,842 & 0,186 \\
\hline 9: Freqüência de manutenção & 0,299 & 0,299 & 0,589 & 0,346 \\
\hline 10: Dificuldade de manutenção & 0,090 & 0,157 & 0,873 & 0,206 \\
\hline \multicolumn{5}{|l|}{ Orgasmo } \\
\hline 11: Freqüência & 0,507 & 0,446 & 0,374 & 0,272 \\
\hline 12: Dificuldade & 0,436 & 0,313 & 0,694 & 0,123 \\
\hline 13: Satisfação & 0,683 & 0,344 & 0,402 & 0,139 \\
\hline \multicolumn{5}{|l|}{ Satisfação } \\
\hline 14: Proximidade com parceiro & 0,726 & 0,249 & 0,427 & 0,180 \\
\hline 15: Com relação sexual & 0,837 & 0,217 & 0,085 & 0,125 \\
\hline 16: Com vida de sexo global & 0,834 & 0,264 & 0,115 & 0,136 \\
\hline \multicolumn{5}{|l|}{ Dor } \\
\hline 17: Freqüência durante penetração & 0,138 & 0,102 & 0,391 & 0,889 \\
\hline 18: Freqüência que segue penetração & 0,181 & 0,196 & 0,125 & 0,837 \\
\hline 19: Grau durante penetração vaginal & 0,121 & 0,162 & 0,383 & 0,895 \\
\hline Percentual de variância explicada & 52,5 & 9,7 & 6,7 & 5,7 \\
\hline Autovalores & 9,97 & 1,85 & 1,27 & 1,08 \\
\hline
\end{tabular}

Nota: em negrito são mostradas as cargas fatoriais maiores que 0,40 .

lação entre lubrificação e orgasmo. Esses resultados estão de acordo com o modelo de resposta sexual no qual o FSFI se baseia 24,25 . Segundo esse modelo proposto por Masters \& Johnson $26 \mathrm{e}$ revisado por outros autores 24 a resposta sexual envolve uma seqüência coordenada de várias fases que inclui desejo, excitação, lubrificação, orgasmo e resolução. Embora o desejo e a excitação não se estabeleçam em uma relação temporal estática, podendo a excitação subjetiva preceder o desejo e vice versa 24 , todos os componentes da resposta são mutuamente dependentes.

Nota-se ainda, baixo índice de correlação entre dor e os demais domínios, o que sugere que a dor no ato sexual possa estar relacionada à ausência dos componentes da resposta sexual.

A análise fatorial demonstrou que não houve concordância com as dimensões teóricas propostas pelos autores para o instrumento original. Na população estudada o instrumento parece medir quatro fatores latentes: desejo/excitação, lubrificação, orgasmo/satisfação e dor.

No presente estudo o fator 1 , com $52,5 \%$ de variância explicada, agrupou os três itens do FSFI associados à satisfação. O agrupamento da questão 6 presente no domínio da excitação deve-se ao fato de que esse item refere-se à satisfação da capacidade de excitar-se e dessa forma trata-se de um componente relacionado com a satisfação da excitação subjetiva. Alguns autores apontam a estreita relação entre orgasmo e satisfação sexual, o que explicaria o agrupamento desses itens no mesmo fator $27,28,29$.

O fator 2 que agrupou os itens dos domínios relativos ao desejo e à excitação também teve um item associado à freqüência do domínio do orgasmo. Esse tipo de agrupamento também ocorreu na análise fatorial do instrumento em inglês, que teve os itens relativos à excitação pontuando também no mesmo fator que orgasmo 4,6. Existe 
Itens constantes do Female Sexual Function Index (FSFI) e resultados do seu exame de consistência interna (alfa de Cronbach total para a escala = 0,948).

\begin{tabular}{|c|c|c|c|c|}
\hline $\begin{array}{l}\text { Domínio (alfa de Cronbach)/ } \\
\text { Questão }\end{array}$ & $\begin{array}{c}\text { Correlação entre } \\
\text { o item e o total } \\
\text { do FSFI }\end{array}$ & $\begin{array}{l}\text { Alfa de Cronbach } \\
\text { total se o item } \\
\text { é desprezado }\end{array}$ & $\begin{array}{l}\text { Correlação entre } \\
\text { o item e o total } \\
\text { do domínio }\end{array}$ & $\begin{array}{l}\text { Alfa de Cronbach } \\
\text { do domínio } \\
\text { se o item é } \\
\text { desprezado }\end{array}$ \\
\hline \multicolumn{5}{|l|}{ Desejo $(0,7867)$} \\
\hline 1: Freqüência & 0,6588 & 0,9462 & - & - \\
\hline 2: Nível & 0,6007 & 0,9472 & - & - \\
\hline \multicolumn{5}{|l|}{ Excitação $(0,8621)$} \\
\hline 3: Freqüência & 0,7399 & 0,9449 & 0,7382 & 0,8121 \\
\hline 4: Nível & 0,7023 & 0,9455 & 0,7177 & 0,8206 \\
\hline 5: Confiança & 0,6442 & 0,9465 & 0,6511 & 0,8476 \\
\hline 6: Satisfação & 0,8029 & 0,9438 & 0,7304 & 0,8153 \\
\hline \multicolumn{5}{|l|}{ Lubrificação $(0,8812)$} \\
\hline 7: Freqüência & 0,7383 & 0,9449 & 0,7121 & 0,8591 \\
\hline 8: Dificuldade & 0,6854 & 0,9458 & 0,7951 & 0,8267 \\
\hline 9: Freqüência de manutenção & 0,7286 & 0,9451 & 0,7119 & 0,8591 \\
\hline 10: Dificuldade de manutenção & 0,6296 & 0,9467 & 0,7505 & 0,8443 \\
\hline \multicolumn{5}{|l|}{ Orgasmo $(0,8575)$} \\
\hline 11: Freqüência & 0,7806 & 0,9442 & 0,7193 & 0,8113 \\
\hline 12: Dificuldade & 0,7242 & 0,9452 & 0,7172 & 0,8133 \\
\hline 13: Satisfação & 0,7821 & 0,9442 & 0,7567 & 0,7761 \\
\hline \multicolumn{5}{|l|}{ Satisfação $(0,8750)$} \\
\hline 14: Proximidade com parceiro & 0,7761 & 0,9443 & 0,7309 & 0,8490 \\
\hline 15: Com relação sexual & 0,6163 & 0,9469 & 0,7565 & 0,8261 \\
\hline 16: Com vida de sexo global & 0,6627 & 0,9462 & 0,7913 & 0,7944 \\
\hline \multicolumn{5}{|l|}{$\operatorname{Dor}(0,9159)$} \\
\hline 17: Freqüência durante penetração & 0,5353 & 0,9483 & 0,8301 & 0,8791 \\
\hline 18: Freqüência que segue penetração & 0,6102 & 0,9470 & 0,8149 & 0,8916 \\
\hline 19: Grau durante penetração vaginal & 0,5578 & 0,9479 & 0,8458 & 0,8661 \\
\hline
\end{tabular}

de fato razão para o agrupamento desses itens, na literatura encontra-se forte correlação entre excitação e orgasmo, de acordo com o modelo de resposta sexual feminina, para haver orgasmo é necessário certo grau de excitação sexual; assim a freqüência de orgasmo estaria relacionada à presença de desejo e excitação sexual 25,30.

$\mathrm{O}$ agrupamento ocorrido no fator 3 incluiu os quatro itens do domínio relativo à lubrificação, com cargas fatoriais maiores que 0,5 , e o item associado à dificuldade/satisfação do domínio do orgasmo. Na resposta sexual da mulher, ausência de lubrificação correlaciona-se com dificuldade de orgasmo 25,30 pois há necessidade de certa congestão pélvica para que este ocorra e a lubrificação vaginal é conseqüência da congestão vascular pélvica.

A dor constituiu-se um domínio à parte dos demais. A presença de dor pode estar relacionada a diversas fases da resposta sexual, contudo não há especificidade nessa relação, isso também é observado no estudo da consistência interna e de correlação inter-domínios e poderia explicar o agrupamento isolado dos três itens do domínio associado à dor no fator 4 .

Essa solução de quatro fatores não quer dizer, porém, que o instrumento não tenha sido adequadamente adaptado, mas isso pode referir-se a uma característica do próprio instrumento. No estudo de validação do questionário original, a análise fatorial também não confirmou a dimensionalidade teórica do FSFI de seis dimensões 4,6. Ao utilizarem o critério dos autovalores mínimos para escolha do número de fatores, os autores encontraram uma solução fatorial de apenas quatro. Eles sugerem, contudo, que um quinto fator teve um autovalor expressivo o suficiente para justificar sua inclusão, mas ainda assim houve confluência dos itens relativos ao desejo e excitação em um mesmo grupo 4,6 . 
Outra possibilidade de explicação para os resultados divergentes das dimensões teóricas do instrumento original é que a resposta sexual feminina é um construto complexo cujas dimensões se correlacionam ${ }^{25}$, sendo difícil distinguilas com clareza. Nessas situações, comuns à grande parte das escalas psicológicas e questionários de medida de satisfação e qualidade de vida, as inter-relações entre os componentes não permitem que os itens apresentem cargas em um único fator 18. Isso aconteceu nas questões 6 e 11 que apresentaram cargas acima de 0,40 nos fatores 1 e 2 e com as questões 12, 13 e 14 que apresentaram cargas mais elevadas nos fatores 1 e 3 .

Além disso, tais instrumentos produzem um primeiro fator composto pela maioria dos itens, explicando a maior parte da variância e outros fatores com menos itens explicando uma menor variância. No presente estudo o fator 1 agrupou sete itens e explica $52,5 \%$ da variância total. Nesse sentido, a interpretação dos resultados de uma análise fatorial deve considerar que a manutenção de componentes no instrumento leve em conta a sua importância teórica no modelo além da solução estatística 18 .

Uma terceira explicação para os resultados da análise fatorial deve-se à população estudada. Um problema comum com o uso de escalas psicológicas é saber se a estrutura fatorial de uma escala pode ser aplicada em amostras com diferentes características sócio-culturais que possam afetar a resposta. Floyd \& Widaman 18 sugerem que nos casos em que há divergência na estrutura fatorial entre grupos, deve-se proceder à análise com uma amostra ampla e não apenas com um subgrupo da população. Contudo, nos casos em que há falha na replicação da estrutura fatorial em grupos com variância similar, há que se decidir sobre a focalização nas variáveis consistentes entre os grupos ou a elaboração de instrumentos específicos para cada subgrupo.

Embora resultados preliminares da amostra utilizada no presente estudo não demonstrem diferença da literatura quanto à freqüência de disfunção sexual, existe clara relação entre nível sócio-econômico e disfunção sexual, sendo sua freqüência maior em estratos sociais mais baixos e em mulheres com menor nível de educação formal ${ }^{1}$. Isso pode configurar a amostra como um subgrupo da população geral devendo-se levar em conta na análise dos resultados a possibilidade de haver diferenças culturais entre esse grupo e a população geral. Essa diferença talvez interfira no entendimento das perguntas ou mesmo dos conceitos sobre os quais o instrumento versa.

Há possibilidade de que a falta de clareza sobre os componentes da resposta sexual possa explicar a convergência entre excitação e desejo, visto que de acordo com o modelo de resposta feminina, excitação sexual subjetiva e desejo podem confundir-se. De outra maneira, as particularidades da população estudada podem também explicar a convergência entre os componentes orgasmo e satisfação. Não é incomum a associação entre orgasmo e satisfação sexual nas mulheres brasileiras, Furlanetto \& Rodrigues 31, em 1996, encontraram tal associação em $80 \%$ das mulheres pesquisadas. A cultura brasileira tende a projetar a relação sexual sem orgasmo como frustrante; para os autores, as mulheres exigem o orgasmo na relação sexual pois o consideram uma vitória 31 .

Todavia, parece que os 19 itens do questionário formam um conjunto consistente no sentido de mensurar um mesmo objeto. A avaliação da consistência interna do FSFI feita através da análise do coeficiente alfa de Cronbach demonstrou uma consistência interna muito alta. Esse resultado é compatível com os estudos de validação do FSFI 4,6,8,9,32 que apresentam valores de alfa de Cronbach para o escore total acima de 0,90. Nota-se ainda que a eliminação de qualquer um dos itens não implica uma significativa mudança do alfa de Cronbach (Tabela 4). Isso é uma evidência de que nenhum item do FSFI estaria mensurando algum aspecto não relacionado ao objeto que pretende ser a resposta sexual feminina. Nota-se, contudo, que os itens 17 e 19, respectivamente a freqüência e o grau da dor durante a penetração vaginal, possuem menor correlação com o total. Isto, porém, não significa que estes itens não sejam úteis na avaliação da resposta sexual feminina, mas é uma evidência de que estejam avaliando tal resposta sob um aspecto diferente dos demais.

Os valores do alfa para cada domínio também indicam uma alta consistência interna em cada componente, ficando em geral acima de 0,85. Apenas o domínio desejo apresentou alfa abaixo de 0,80 . Isso poderia ser outra evidência de que o domínio desejo não se configure como um domínio isolado, mas associado à excitação.

Contudo, a recente proposta de avaliação da excitação feminina considera a excitação subjetiva e o desejo como dimensões distintas da resposta sexual feminina muito embora reconheça a expressão da excitação tanto no âmbito periférico (lubrificação vaginal) quanto central. É pretensão do FSFI mensurar distintamente desejo e excitação, mas essa separação parece ser mais teórica que estatística.

O FSFI apresenta outras vantagens, pois é conciso, capaz de ser administrado em um curto período de tempo e com capacidade de identificar mudanças após intervenção. Desde sua pu- 
blicação ele tem sido adaptado para outras línguas e vários estudos baseados nessas versões estão publicados ou em andamento. Exemplo disso são os estudos de Blümel et al. ${ }^{8}$ que adaptaram o índice para o contexto cultural chileno e os estudos de ter Kuile et al. ${ }^{9}$ que avaliaram as propriedades psicométricas do instrumento na população alemã.

No Brasil, ainda não havia método com essas características desenvolvido em língua portuguesa, ou adaptado a esse contexto cultural, que pudesse ser utilizado em larga escala em estudos epidemiológicos. Abdo et al. 13 conduzem estudos de base populacional, e avaliam a presença de problemas e dificuldades sexuais. Recentemente Abdo 14 propôs um índice numérico para aumentar o poder de análise de estudos como esses.

Contudo, a adaptação e validação de um mesmo instrumento para vários idiomas é desejável 33 , pois traz a possibilidade de se obter dados possíveis de comparação entre culturas e países diferentes. Por esse motivo, outros estudos foram realizados com a finalidade de apresentar uma versão em português para o FSFI.

Leite et al. 34, em 2007, apresentam resultado de estudo de tradução e aplicação da versão do FSFI em população de mulheres grávidas. Os autores, embora façam referência à validade de construto, apresentam um alfa de Cronbach para cada domínio acima de 0,79 , e testam a validade de critério fazendo uma comparação do FSFI com um outro instrumento de medida da função sexual. Contudo, apenas considerando a população estudada (gestantes no terceiro trimestre de gravidez) que apresenta uma forte alteração na resposta sexual quando comparada à população geral 35, não se poderia comparar os resultados com estudos realizados em outras populações.

Em 2008, outro artigo apresenta os resultados da tradução e aplicação de uma versão do FSFI em população de mulheres que buscam tratamento para infertilidade e mulheres que buscam esterilização feminina 36 . Porém, os autores utili- zam um modelo de tradução que não apresenta referência na literatura, e não descrevem os detalhes do processo de tradução. Além disso, esses consideram válida a versão somente a partir da análise do índice de correlação de Pearson e do coeficiente alfa de Cronbach para o valor total do índice. Embora se observe no estudo valor alfa de 0,62 para o domínio desejo, os autores consideram os valores de alfa total acima de 0,90 suficientes para propor a utilização do instrumento na população brasileira.

Observa-se também que nenhum desses estudos apresenta uma análise fatorial como parte do processo de validação de construto, que segundo alguns autores 17,18,19,20 é um procedimento necessário para a validação de instrumentos de medida psicológica e de qualidade de vida. O que se aponta é que embora negligenciado em muitos artigos, o processo de validação de construto, que inclui a análise fatorial, é de extrema importância para a definição das propriedades psicométricas de um instrumento de medida.

\section{Conclusão}

No presente estudo o questionário FSFI mostrou-se culturalmente adequado, compreensível e com um baixo índice de não resposta $(1,7 \%)$ na aplicação face a face. Porém as análises estatísticas apontam para uma discordância com as dimensões teóricas do instrumento original. Isso pode ser devido a uma característica do instrumento ou pode apontar a necessidade de revisão dos fatores latentes do questionário. Contudo a homogeneidade da população estudada quanto à renda, educação e nível sócio-econômico não permite que se façam proposições para a adequação da estrutura do FSFI. Há necessidade de desenvolver outros estudos na população geral para melhor definição do conjunto de propriedades do FSFI, como sua composição de fatores latentes e o estabelecimento de pontos de corte adequados à população brasileira. 


\section{Resumo}

Este trabalho teve como objetivos avaliar a validade de construto de uma versão do Female Sexual Function Index (FSFI). A versão foi inserida ao final de um questionário multidimensional e aplicada face a face em 235 mulheres esterilizadas. $O$ índice de não resposta foi de 1,7\%. A amostra apresentou-se bastante homogênea com baixa renda e nível educacional. Um coeficiente alfa de Cronbach de 0,948 indicou uma consistência interna muito alta. A análise fatorial demonstrou que o instrumento parece medir quatro fatores latentes: desejo/excitação, lubrificação, orgasmo/satisfação e dor. Isso pode referir-se a uma característica do próprio instrumento, às características da resposta sexual feminina ou ainda às peculiaridades culturais da amostra, o que pode interferir no entendimento das perguntas ou dos conceitos sobre os quais o instrumento versa. Assim aponta-se para necessidade de desenvolvimento de outros estudos na população geral para a definição do conjunto de propriedades do FSFI, como sua composição de fatores latentes e o estabelecimento de pontos de corte adequados à população brasileira.

Sexualidade; Questionários; Saúde da Mulher

\section{Referências}

1. Laumann EO, Paik A, Rosen RC. Sexual dysfunction in the United States: prevalence and predictors. JAMA 1999; 281:537-44.

2. Basson R, Berman J, Burnett A, Derogatis L, Ferguson D, Fourcroy J, et al. Report of the international consensus development conference on female sexual dysfunction: definitions and classifications. J Urol 2000; 163:888-93.

3. Althof SE, Rosen RC, Derogatis L, Corty E, Quirk F, Symonds T. Outcome measurement in female sexual dysfunction clinical trials: review and recommendations. J Sex Marital Ther 2005; 31:153-66.

4. Rosen R, Brown C, Heiman J, Leiblum S, Meston C, Shabsigh R, et al. The Female Sexual Function Index (FSFI): a multidimensional self-report instrument for the assessment of female sexual function. J Sex Marital Ther 2000; 26:191-208.

5. Meston CM, Derogatis LR. Validated instruments for assessing female sexual function. J Sex Marital Ther 2002; 28 Suppl 1:155-64.

\section{Colaboradores}

R. C. Pacagnella adaptou o instrumento, fez sua validação analisando os dados sob orientação de E. M. Vieira que coordenou o projeto de pesquisa, desenhou o estudo e participou da análise de dados. E. Z. Martinez orientou a análise de dados apresentada neste artigo. Os três autores participaram igualmente da elaboração deste manuscrito.

\section{Agradecimentos}

Este estudo foi financiado pela Fundação de Amparo à Pesquisa do Estado de São Paulo (FAPESP; processo no. 03/0549) e serviu de base para a Dissertação de Mestrado do primeiro autor.
6. Wiegel M, Meston C, Rosen R. The Female Sexual Function Index (FSFI): cross-validation and development of clinical cutoff scores. J Sex Marital Ther 2005; 31:1-20.

7. Rellini A, Meston C. The sensitivity of event logs, self-administered questionnaires and photoplethysmography to detect treatment-induced changes in female sexual arousal disorder (FSAD) diagnosis. J Sex Med 2006; 3:283-91.

8. Blümel JEM, Binfa LE, Cataldo PA, Carrasco AV, Izaguirre HL, Sarrá SC. Indice de función sexual femenina: un test para evaluar la sexualidad de la mujer. Rev Chil Obstet Ginecol 2004; 69:118-25.

9. ter Kuile MM, Brauer M, Laan E. The Female Sexual Function Index (FSFI) and the Female Sexual Distress Scale (FSDS): psychometric properties within a Dutch population. J Sex Marital Ther 2006; 32:289-304.

10. Penteado SR, Fonseca AM, Bagnoli VR, ASSIS J, Pinotti JA. Avaliação da capacidade orgástica em mulheres na pós-menopausa. Rev Assoc Med Bras 2008; 50:444-50. 
11. Fonseca AM, Bagnoli VR, Penteado SR, Paixao JS, Cavalcanti AL, Pinotti JA. Monophasic estrogenprogestogen therapy and sexuality in postmenopausal women. Clin Drug Investig 2007; 27:131-7.

12. Cavalcanti AL, Bagnoli VR, Fonseca AM, Pastore RA, Cardoso EB, Paixao JS, et al. Effect of sildenafil on clitoral blood flow and sexual response in postmenopausal women with orgasmic dysfunction. Int J Gynaecol Obstet 2008; 102:115-9.

13. Abdo CHN, Moreira Junior ED, Fittipaldi JAS. Estudo do comportamento sexual no Brasil. RBM Rev Bras Med 2000; 57:1329-35.

14. Abdo CHN. Elaboração e validação do quociente sexual: versão feminina: uma escala para avaliar a função sexual da mulher. RBM Rev Bras Med 2006; 63:477-82.

15. Pacagnella RC, Vieira EM, Rodrigues Jr. OM, Souza C. Adaptação transcultural do Female Sexual Function Index. Cad Saúde Pública 2008; 24:416-26.

16. Falcão DM, Ciconelli RM, Ferraz MB. Translation and cultural adaptation of quality of life questionnaires: an evaluation of methodology. J Rheumatol 2003; 30:379-85.

17. Guillemin F, Bombardier C, Beaton D. Cross-cultural adaptation of health-related quality of life measures: literature review and proposed guidelines. J Clin Epidemiol 1993; 46:1417-32.

18. Floyd F, Widaman K. Factor analisys in the development and refinement of clinical assessment instruments. Psychol Assess 1995; 7:286-99.

19. Guillemin F. Cross-cultural adaptation and validation of health status measures. Scand J Rheumatol 1995; 24:61-3.

20. Pasquali L. Psicometria: teoria dos testes na psicologia e na educação. Petrópolis: Editora Vozes; 2003.

21. Vieira EM. Avaliação da oferta de métodos cirúrgicos no planejamento familiar em Ribeirão Preto, SP [Tese de Livre Docência]. Ribeirão Preto: Faculdade de Medicina de Ribeirão Preto, Universidade de São Paulo; 2006.

22. Artes R. Aspectos estatísticos da análise fatorial de escalas de avaliação. Rev Psiquiatr Clín (São Paulo) 1998; 25:223-8.
23. Rosen RC. Assessment of female sexual dysfunction: review of validated methods. Fertil Steril 2002; 77 Suppl 4:S89-93.

24. Basson R. Recent advances in women's sexual function and dysfunction. Menopause 2004; 11 (6 Pt 2):714-25.

25. Basson R, Leiblum S, Brotto L, Derogatis L, Fourcroy J, Fugl-Meyer K, et al. Revised definitions of women's sexual dysfunction. J Sex Med 2004; 1:40-8.

26. Masters WH, Johnson VE. Human sexual response. Toronto/New York: Bantam Books; 1966.

27. Haavio-Mannila E, Kontula O. Correlates of increased sexual satisfaction. Arch Sex Behav 1997; 26:399-419.

28. Mah K, Binik YM. The nature of human orgasm: a critical review of major trends. Clin Psychol Rev 2001; 21:823-56.

29. Young M, Denny G, Luquis R, Young T. Correlates of sexual satisfaction in marriage. Can J Hum Sex 1998; 7:115-27.

30. Basson R, Brotto LA, Laan E, Redmond G, Utian WH. Assessment and management of women's sexual dysfunctions: problematic desire and arousal. J Sex Med 2005; 2:291-300.

31. Furlanetto S, Rodrigues Jr. O. A satisfação sexual da mulher adulta. Rev Bras Sex Hum 1996; 7:131-43.

32 Meston CM. Validation of the Female Sexual Function Index (FSFI) in women with female orgasmic disorder and in women with hypoactive sexual desire disorder. J Sex Marital Ther 2003; 29:39-46.

33. Jones LRA. The use of validated questionnaires to assess female sexual dysfunction. World J Urol 2002; 20:89-92.

34. Leite APL, Moura EA, Campos AAS, Mattar R, Souza E, Camano L. Validação do índice da função sexual feminina em grávidas brasileiras. Rev Bras Ginecol Obstet 2007; 29:396-401.

35. Read J. Sexual problems associated with infertility, pregnancy, and ageing. BMJ 2004; 329:559-61.

36. Hentschel H, Alberton DL, Capp E, Goldim JR, Passos EP. Validação do Female Sexual Function Index (FSFI) para uso em língua portuguesa. Rev HCPA \& Fac Med Univ Fed Rio Gd do Sul 2008; 27:127-33.

Recebido em 15/Set/2008

Versão final reapresentada em 15/Mai/2009

Aprovado em 10/Jul/2009 